\title{
Impact of pulmonary vein isolation on obstructive sleep apnea in patients with atrial fibrillation
}

\author{
Friedrich Felix Hoyer, Katharina Henrich, Jens Kreuz, Carmen Pizarro, \\ Jan-Wilko Schrickel, Lars Martin Lickfett, Erica Mittmann-Braun, \\ Uwe Juergens, Georg Nickenig, Dirk Skowasch
}

Department of Internal Medicine II, Cardiology, Pneumology, University of Bonn, Germany

\begin{abstract}
Background: Obstructive sleep apnea (OSA) has been identified as associated with the onset and propagation of atrial fibrillation $(A F)$ and predicts recurrences of $A F$ after pulmonary vein isolation (PVI). Vice versa, it has never been investigated whether PVI influences OSA. However, it has been controversial whether a restored atrial function can affect the course of OSA. Therefore, we have assessed whether PVI procedure modulates the prevalence and severity of OSA.
\end{abstract}

Methods and Results: We included 23 individuals with AF that were assigned to undergo PVI into this study. Patients were $65 \pm 7$ years old, obese $\left(B M I 29.9 \pm 5.4 \mathrm{~kg} / \mathrm{m}^{2}\right)$, white (100\%) and had a normal left ventricular function (LVEF $64 \pm 9 \%$ ). Polygraphic assessment was carried out before and 6 months after PVI. The prevalence of OSA, defined as an apneahypopnea index (AHI) $\geq 5$ per hour of sleep, was $74 \%$ before PVI compared to $70 \% 6$ months after the procedure ( $p>0.05$ ). Severity of OSA did not differ (AHI before vs. after: $18 \pm 18 / \mathrm{h}$ vs. $15 \pm 17 / h, p>0.05)$ as well as further polygraphic parameters did not differ before and after the procedure.

Conclusions: Prevalence and severity of OSA are not affected by PVI in patients suffering from AF. (Cardiol J 2014; 21, 4: 392-396)

Key words: obstructive sleep apnea, pulmonary vein isolation, atrial fibrillation

\section{Introduction}

Atrial fibrillation $(\mathrm{AF})$ remains the most frequent arrhythmia occurring in clinical practice [1]. Serious short- and long-term sequelae such as thrombembolic stroke, heart failure, reduced quality of life and increased overall mortality ask for further exploration of this disorder [2]. While several underlying disorders such as coronary artery disease and hypertension have been identified to be closely connected with the onset of this disease, there is still an enormous rate of $\mathrm{AF}$ that appears without co-existing classical cardiovascular conditions.
Therefore, further unknown instigating factors triggering and propagating $\mathrm{AF}$ have been assumed. One risk factor that has previously been shown to be independently associated with the onset of AF is obstructive sleep apnea (OSA) [3]. OSA is initiated by upper airway obstruction that is physiologically responded by augmented respiratory efforts, thereby increasing the intrathoracic pressure that finally leads to increased atrial vulnerability and atrial chamber enlargement. It has been suggested that these mechanisms also induce tissue remodeling at the pulmonary veins that are considered to provide arrhythmogenic areas inducing AF by electrical

Address for correspondence: Dr Friedrich Felix Hoyer, Department of Internal Medicine II, Cardiology, University of Bonn, Sigmund-Freud-Str. 25, 53127 Bonn, Germany, tel: +49 228287 51487, fax: +49 22828751480 ,

e-mail: Felix.Hoyer@ukb.uni-bonn.de

Received: 26.06.2013 Accepted: 25.07.2013 
avalanches [1]. Pulmonary vein isolation (PVI) diminishes the occurrence of $\mathrm{AF}$ by interrupting these electrical lines and usually leads to a significant impairment of AF burden [4-7]. Further, it can also induce reverse atrial remodeling [8]. Almost one-third of the patients undergoing PVI does not benefit from this procedure $[5,9]$. Recently, we have shown that the prevalence and severity of OSA is particularly high in this group of patients that show resistance to PVI [10]. Our finding was recently corroborated by a study yielding that moderate to severe sleep-disordered breathing disturbances independently predict recurrences of AF after PVI as well [11]. To date, though various studies investigated the effects of OSA on AF, it has never been contemplated whether PVI might impact on the severity of OSA vice versa. However, it has been speculated that AF with subsequent increased left-atrial pressure can lead to pulmonary congestion and thereby might cause and aggravate OSA [12]. Interestingly, Garrigue et al. showed that atrial overdrive pacing diminished the amount of both central and OSA in patients with a permanent pacemaker implanted for sinus node dysfunction [13]. Given the vast number of studies concerning OSA and AF, this question should be addressed in order to unravel unknown factors possibly skewing the relation between OSA and PVI. Therefore, we conducted a pilot study to determine the prevalence and severity of OSA in patients with non-permanent AF before PVI and 6 months after this procedure.

\section{Methods}

\section{Patients}

Patients with non-permanent AF that were assigned to undergo PVI were consecutively enrolled in this study. Patients were excluded if they had reduced left ventricular ejection fraction, permanent AF, a history of poor compliance with drug treatment, evidence of the use of arrhythmogenic substances such as ethanol, cocaine and 1-thyroxin, or known OSA with or without continuous positive airway pressure treatment. Twenty-three patients that were analyzed before and after PVI were generally late middle-aged (65 \pm 7 years old), white (100\%), and overweight $29.9 \pm 5.4 \mathrm{~kg} / \mathrm{m}^{2}$ (Table 1 ). This study was approved by the local ethics committee and carried out in accordance with the declaration of Helsinki. Written informed consent was obtained from every individual.
Table 1. Baseline demographic and clinical characteristics of the patients.

\begin{tabular}{lc}
\hline Variable & $\mathbf{N}=23$ \\
\hline Persistent AF & $13(57 \%)$ \\
Age [years] & $65 \pm 7$ \\
Male sex & $16(70 \%)$ \\
BMI $\left[\mathrm{kg} / \mathrm{m}^{2}\right]$ & $29.9 \pm 5.4$ \\
LVEF $[\%]$ & $64 \pm 9$ \\
CAD & $4(7 \%)$ \\
Diabetes & $3(13 \%)$ \\
Hypertension & $16(70 \%)$ \\
Creatinin [mg/dL] & $1.1 \pm 0.4$ \\
ACEl & $5(22 \%)$ \\
Beta-blocker & $17(74 \%)$ \\
Statin & $9(39 \%)$ \\
Diuretic & $5(22 \%)$ \\
Amiodarone & $2(8 \%)$ \\
Dronedaron & $5(22 \%)$ \\
Class 1c & $7(30 \%)$ \\
Digitalis & $4(17 \%)$ \\
Ca ${ }^{2+}$-antagonist & $7(30 \%)$ \\
Aspirin & $4(17 \%)$ \\
Marcumar & $22(96 \%)$ \\
\hline AF &
\end{tabular}

$\mathrm{AF}$ - atrial fibrillation; BMI — body mass index; LVEF — left ventricular ejection fraction; CAD - coronary artery disease; ACEI - angiotensin converting enzyme inhibitor

\section{Polygraphic assessment}

All patients underwent an overnight polygraphic study the day before PVI was performed and 6 months after the procedure. Conventional polygraphic devices were used (Poly MESAM Device, ResMed). During the polygraphic examination, heart rhythm was determined using a precordial electrocardiograph. Thoraco-abdominal movements were assessed by a calibrated respiratory conductance phlethysmograph and oxygen saturation was measured by fingertip oximetry. The mean lowest oxygen saturation $\left(\mathrm{SaO}_{2}\right)$ during sleep was calculated by averaging the lowest $\mathrm{SaO}_{2}$ for each $30 \mathrm{~s}$ episode during sleep. OSA was defined as an absence of airflow for at least $10 \mathrm{~s}$ and hypopneas were defined as a reduction in airflow for more than $50 \%$ from the baseline level for at least $10 \mathrm{~s}$ with $3 \%$ decrease in oxygen saturation while paradoxical thoraco-abdominal movements occurred. Subjects with more than 5 obstructive apneas and hypopneas were considered to suffer from OSA according to present guidelines [14, 15]. Analysis and interpretation of the obtained data 
were performed blinded without knowledge about the patient's status.

\section{Ablation procedure}

PVI was performed by cryoballoon ablation technique as previously described [16]. Ablation success was controlled again $20 \mathrm{~min}$ after the last pulmonary vein was isolated. To prevent shortterm recurrences of $\mathrm{AF}$, patients were continued on prescribed anti-arrhythmic medication they had at the time of referral for PVI for further 3 months.

\section{Follow-up}

The follow-up period began immediately on the day after procedure and was continued at least until the second polygraphic study was performed. Patients were equipped with an event recorder and instructed to transmit one electrocardiogram (ECG) per day and to carry out additional measurements if they felt any symptoms of AF. Standard duration of all records was 1 min. Recording started 1 day after PVI and was continued for further 3 months. All recordings were analyzed for the occurrence of AF. The first month after the procedure was not included into the analysis and all episodes of $\mathrm{AF}$ within that month were therefore excluded. Patients were examined again 3 months after the procedure in our outpatients' department. Furthermore, a detailed medical history and a 12-lead ECG were taken. Patients were then followed up on a regular basis monthly. Routine and Holter ECG were performed regardless of symptoms. If asymptomatic AF was detected on conventional ECG or event recorder, patients were considered suffering from recurrent AF. If no episode of AF could be detected with the different applied instruments until the second polygraphic study was performed, patients were considered to be successfully treated by PVI.

\section{Statistical analysis}

Continuous variables were expressed as mean \pm standard deviation and categorial variables were expressed in percentages. Continuous data were analyzed by means of unpaired students' t-test and categorial variables were analyzed using $\chi^{2}$ test. Statistical analysis was carried out with SPSS (Statistical Package for Social Sciences, Version 17, Chicago, IL, USA). $\mathrm{P}<0.05$ was considered to be statistically significant.

\section{Results}

Individuals suffering from $\mathrm{AF}(\mathrm{n}=23)$ were enrolled into this arm of the study. Polygraphic as- sessment was performed before PVI and 6 months afterwards. OSA was detected in $74 \%$ of the patients before PVI was performed and in $70 \%$ of the patients 6 months after PVI ( $p>0.05)$, indicating that PVI did not influence the prevalence of OSA in these patients. The overall severity of OSA in this population, as expressed by the apnea-hypopnea index (AHI), did not differ as well (AHI events/h: before $18 \pm 18$ vs. after $15 \pm 17, \mathrm{p}>0.05$ ) (Table 2 ). Assessment of the oxygen desaturation index (ODI) did not reveal any differences (ODI events/h: before $13 \pm 12$ vs. $13 \pm 14$, p > 0.05). Other polygraphic parameters did not differ before and after PVI as well.

Fifteen patients remained in sinus rhythm and were considered to be successfully treated by PVI while 8 patients suffered from recurrent $\mathrm{AF}$ after PVI during the follow-up period. Prevalence of OSA in patients that were cured from $\mathrm{AF}$ was $80 \%$ before PVI compared to $73 \%$ after PVI (before $80 \%$ vs. after $73 \%, \mathrm{p}>0.05$ ). Again, we did not detect any differences with regards to the severity of OSA in successfully treated patients (AHI events/h: before $20 \pm 18$ vs. after $16 \pm 12, p>0.05$ ). Assessment of the ODI did not reveal any differences as well (ODI events/h: before $14 \pm 12$ vs. $15 \pm 11, \mathrm{p}>0.05$ ). Relevant central sleep apnea was not detected in the study population.

\section{Discussion}

We have analyzed the effects of PVI procedure in patients with AF on the course of OSA. The possibility that OSA can be affected by PVI has not been ruled out yet, though it is conceivable that successfully treating $\mathrm{AF}$ associated with a restored atrial function might also modulate the prevalence and severity of OSA in these patients. Here, we provide first evidence demonstrating that PVI does neither affect the prevalence nor the severity of OSA in patients undergoing PVI. Considering the large number of investigations studying the effects of OSA on AF [17], this finding is an important contribution to the scientific community that is necessary for a better interpretation of the association of OSA and AF. It has recently been demonstrated that renal sympathetic denervation does not only lower blood pressure but is also accompanied by an improvement of OSA indicated by a diminishment in AHI in patients suffering from therapyresistant hypertension [18]. Although the exact mechanism has not been identified in this study, it is conceivable that the reduced sympathetic tonus and reduced fluid overload are responsible for the observed improvement in OSA. 
Table 2. Results of polygraphic assessment. Prevalence of obstructive sleep apnea (OSA) and mean apnea-hypopnea index (AHI) - before and 6 months after pulmonary vein isolation (PVI). Results of all individuals $(n=23)$ are shown in the upper part. Results of patients $(n=15)$ that were successfully treated by PVI are shown in the lower part.

\begin{tabular}{|c|c|c|c|}
\hline All & $\begin{array}{c}\text { Before PVI } \\
(\mathrm{n}=23)\end{array}$ & $\begin{array}{c}\text { After PVI } \\
(\mathrm{n}=23)\end{array}$ & $\mathbf{P}$ \\
\hline Prevalence OSA & $74 \%$ & $70 \%$ & 0.67 \\
\hline AHI [events/h] & $18 \pm 18$ & $15 \pm 17$ & 0.63 \\
\hline ODI [events/h] & $13 \pm 12$ & $13 \pm 14$ & 0.97 \\
\hline Mean $\mathrm{SaO}_{2}[\%]$ & $92 \pm 4$ & $93 \pm 4$ & 0.49 \\
\hline Minimal $\mathrm{SaO}_{2}[\%]$ & $74 \pm 24$ & $81 \pm 17$ & 0.26 \\
\hline ESS score & $8 \pm 3$ & $9 \pm 5$ & 0.40 \\
\hline Heart rate $[\mathrm{bpm}]$ & $75 \pm 25$ & $66 \pm 10$ & 0.49 \\
\hline AF-free group & $\begin{array}{c}\text { Before PVI } \\
(\mathrm{n}=15)\end{array}$ & $\begin{array}{c}\text { After PVI } \\
(n=15)\end{array}$ & $\mathbf{P}$ \\
\hline Prevalence OSA & $80 \%$ & $73 \%$ & 0.74 \\
\hline AHI [events/h] & $20 \pm 18$ & $16 \pm 12$ & 0.53 \\
\hline ODI [events/h] & $14 \pm 12$ & $15 \pm 11$ & 0.91 \\
\hline Mean $\mathrm{SaO}_{2}[\%]$ & $92 \pm 3$ & $93 \pm 3$ & 0.40 \\
\hline Minimal $\mathrm{SaO}_{2}[\%]$ & $75 \pm 22$ & $83 \pm 8$ & 0.23 \\
\hline ESS score & $8 \pm 3$ & $11 \pm 5$ & 0.12 \\
\hline Heart rate $[\mathrm{bpm}]$ & $77 \pm 30$ & $66 \pm 10$ & 0.19 \\
\hline
\end{tabular}

Chi-square test and unpaired t-test were used for statistical analysis. Data are mean \pm standard deviation; AF — atrial fibrillation; ODI — oxygen-desaturation index; $\mathrm{SaO}_{2}$ - oxygen saturation; ESS — Epworth Sleepiness Scale

There have been controversial studies investigating the effects of atrial function on OSA. Garrigue et al. reported a reduction of sleep-disordered breathing in patients that received a pacemaker with atrial overdrive pacing in order to reduce the incidence of atrial tachyarrhythmias [13]. Interestingly, the authors did not only report a reduction of central sleep apnea but - unexpectedly — also of OSA. However, the mechanism implicated in that improvement remained unclear. In our study, we investigated the effect of therapeutic PVI on OSA.

Although the majority of individuals was cured from AF burden and remained in sinus rhythm after PVI, we did not detect any improvements pertaining to AHI. If there was an effect of PVI on OSA, one would expect an improvement of OSA especially in patients that were cured from AF. However, we detected sleep profile differences neither in the overall study population nor selectively in the group of patients that were cured after PVI. Although the prevalence of OSA was relatively high in patients that were successfully treated via PVI, one may speculate that these individuals are less prone to OSA-induced recurrent AF. On the other hand, it has recently been shown that electrical remodeling particularly occurs in patients with more severe forms of OSA (AHI > 30 events/h) [19] while our population suffered from moderate OSA. Nonetheless, our finding suggests that neither a restored left atrial function nor PVI, respectively, impact on OSA. Another study is in line with our finding. Krahn's study showed that temporary atrial pacing does not appear to improve respiratory manifestations of OSA [13].

\section{Limitations of the study}

This study has several limitations that should be considered. (1) The number of patients involved in this study is low. Larger trials have to corroborate the results obtained in this analysis. (2) PVI can influence left atrial remodeling and even induce reverse atrial remodeling [8] that can be assessed via echocardiography. For this study we did not obtain any echocardiographic parameters of the left atrium. However, echocardiography would allow a better assessment of atrial function and several atrial parameters. (3) Further, no electrical assessment of atrial remodeling, such as signal-averaged p-wave duration, was obtained. Therefore, further studies are needed to selectively correlate the course of OSA after PVI with features of structural and electrical atrial remodeling. (4) We have ana- 
lyzed patients with non-permanent $\mathrm{AF}$ that were scheduled to undergo PVI. It has to be mentioned that the effect of PVI on restoring atrial function is less in this population compared to patients suffering from long lasting AF. (5) Finally, our population suffered from moderate OSA. However, it has been shown that particularly severe forms of OSA influence atrial remodeling. Thus, if PVI influences sleep parameters, it is conceivable that these effects are only detectable in more severe forms of OSA in which atrial remodeling is farther progressed before PVI is performed.

\section{Conclusions}

PVI in patients suffering from AF does not influence the prevalence and severity of OSA.

\section{Acknowledgements}

The authors gratefully acknowledge the expert technical assistance of Karin Springmann.

\section{Conflict of interest: none declared}

\section{References}

1. Fuster V, Rydén LE, Cannom DS et al. ACC/AHA/ESC 2006 Guidelines for the Management of Patients with Atrial Fibrillation: A report of the American College of Cardiology/American Heart Association Task Force on Practice Guidelines and the European Society of Cardiology Committee for Practice Guidelines (Writing Committee to Revise the 2001 Guidelines for the Management of Patients With Atrial Fibrillation): Developed in collaboration with the European Heart Rhythm Association and the Heart Rhythm Society. Circulation, 2006. 114(7): p. e257-354.

2. Benjamin EJ, Wolf PA, D‘Agostino RB, Silbershatz H, Kannel WB, Levy D. Impact of atrial fibrillation on the risk of death: The Framingham Heart Study. Circulation, 1998; 98: 946-952.

3. Gami AS, Pressman G, Caples SM et al. Association of atrial fibrillation and obstructive sleep apnea. Circulation, 2004; 110: 364-367.

4. Haissaguerre M, Jaïs P, Shah DC et al. Spontaneous initiation of atrial fibrillation by ectopic beats originating in the pulmonary veins. N Engl J Med, 1998; 339: 659-666.

5. Pappone C, Oreto G, Rosanio S et al. Atrial electroanatomic remodeling after circumferential radiofrequency pulmonary vein ablation: efficacy of an anatomic approach in a large cohort of patients with atrial fibrillation. Circulation, 2001; 104: 2539-2544.

6. Cappato R, Calkins H, Chen SA et al. Worldwide survey on the methods, efficacy, and safety of catheter ablation for human atrial fibrillation. Circulation, 2005; 111: 1100-1105.
7. Bertaglia E, Scarabeo V, Zoppo F et al. [Circumferential isolation of pulmonary veins with transcatheter radiofrequency ablation in the treatment of atrial fibrillation]. Ital Heart J Suppl, 2003; 4: 825-832.

8. Pang H, Ronderos R, Pérez-Riera AR, Femenía F, Baranchuk A. Reverse atrial electrical remodeling: a systematic review. Cardiol J, 2011; 18: 625-631.

9. Ernst S, Ouyang F, Löber F, Antz M, Kuck KH. Catheter-induced linear lesions in the left atrium in patients with atrial fibrillation: An electroanatomic study. J Am Coll Cardiol, 2003; 42: 1271-1282.

10. Hoyer FF, Lickfett LM, Mittmann-Braun E et al. High prevalence of obstructive sleep apnea in patients with resistant paroxysmal atrial fibrillation after pulmonary vein isolation. J Interv Card Electrophysiol, 2010; 29: 37-41.

11. Bitter T, Nölker G, Vogt J, Prinz C, Horstkotte D, Oldenburg O. Predictors of recurrence in patients undergoing cryoballoon ablation for treatment of atrial fibrillation: The independent role of sleep-disordered breathing. J Cardiovasc Electrophysiol, 2012; 23: $18-25$.

12. Stevenson IH, Teichtahl H, Cunnington D, Ciavarella S, Gordon I, Kalman JM. Prevalence of sleep disordered breathing in paroxysmal and persistent atrial fibrillation patients with normal left ventricular function. Eur Heart J, 2008; 29: 1662-1669.

13. Lamba J, Simpson CS, Redfearn DP, Michael KA, Fitzpatrick M, Baranchuk A. Cardiac resynchronization therapy for the treatment of sleep apnoea: A meta-analysis. Europace, 2011; 13: 1174-1179.

14. Somers VK, White DP, Amin R et al. Sleep apnea and cardiovascular disease: An American Heart Association/american College Of Cardiology Foundation Scientific Statement from the American Heart Association Council for High Blood Pressure Research Professional Education Committee, Council on Clinical Cardiology, Stroke Council, and Council On Cardiovascular Nursing. In collaboration with the National Heart, Lung, and Blood Institute National Center on Sleep Disorders Research (National Institutes of Health). Circulation, 2008; 118: 1080-1111.

15. Ruehland WR, Rochford PD, O'Donoghue FJ, Pierce RJ, Singh P, Thornton AT. The new AASM criteria for scoring hypopneas: impact on the apnea hypopnea index. Sleep, 2009; 32: 150-157.

16. Linhart M, Bellman B, Mittmann-Braun E et al. Comparison of cryoballoon and radiofrequency ablation of pulmonary veins in 40 patients with paroxysmal atrial fibrillation: A case-control study. J Cardiovasc Electrophysiol, 2009; 20: 1343-1348.

17. Digby GC. Sleep apnea and atrial fibrillation; 2012 update. Curr Cardiol Rev, 2012; 8: 265-272.

18. Witkowski A, Prejbisz A, Florczak E et al. Effects of renal sympathetic denervation on blood pressure, sleep apnea course, and glycemic control in patients with resistant hypertension and sleep apnea. Hypertension, 2011; 58: 559-565.

19. Baranchuk A, Pang H, Seaborn GE et al. Reverse atrial electrical remodelling induced by continuous positive airway pressure in patients with severe obstructive sleep apnoea. J Interv Card Electrophysiol, 2013; 36: 247-253. 